\title{
Etude globale pour une stratégie de réduction des risques dus aux crues du Rhône
}

\section{Global study for a strategy of risk reduction due to Rhone river floods}

\author{
par E. Guillaumin, B. Cortier
}

Institution interdépartementale des bassins Rhône Saône.

\author{
L. Levasseur
}

Compagnie nationale du Rhône.

\author{
P. Michelet
}

Agence de l'Eau Rhône Méditerranée Corse.

J.-B. Le Hy, J. Dumez

DIREN Rhône Alpes, Délégation de bassin Rhône Méditerranée Corse.

The river is characterised by complex hydrological and hydraulic regimes. It is fed by a large number of tributaries with strong flood flows, draining a valley dominated by mountainous massifs and varying climatic conditions. As for the Rhone Valley, it is highly urbanised with large concentrations of industry. In addition, the operation of the river has been modified considerably by developments constructed for hydropower, agricultural and navigation requirements. Nonetheless, the diversity of the environments through which it flows still makes the Rhone a rich fund of habitats and species of flora and fauma, remarkable both in France and Europe. Lastly, whether superficial or underground (alluvial water), the waters of the Rhone constitute a key factor for the valley's development.

Given the extent of these challenges, the Catchment Area Committee adopted a "Rhone action plan" in 1992, after carrying out a major study programme. This aimed at restoring the quality of the components making up the hydrosystem (water quality, by-passed sections and environments associated with the river, migration plans, etc.). In order to take new knowledge into account, the Rhone-Mediterranean-Corsica SDAGE, adopted in 1996 by the Catchment Area Committee, programmes the updating of this action plan.

Following the heavy flooding of 1993 and 1994, which caused major damage along the Rhone, the Ministry of the Environment decided to make an overall study of the operation of the river under flood conditions, taking into account all the developments constructed over the last fifty years. The objective announced was to carry a wide-ranging study covering the entire catchment area, in order to modify current land use practices in flood plains and reinforce the protection of urban areas against floods. The promoter plans to carry out wide-ranging consultation with neighbouring populations, etc., in order to define a strategy to reduce the risks of floods along the Rhone.

\section{I INTRODUCTION}

Les crues de 1993 et de 1994 ont été un véritable choc pour la vallée du Rhône ; l'absence de crues fortes durant une longue période ainsi que les travaux d'endiguement de la CNR ont fait croire à tort aux riverains qu'ils étaient désormais à l'abri des inondations.

Le coût élevé des dommages occasionnés par les inondations a mis en évidence la nécessité de rechercher de nouveaux modes de gestion et d'aménagement du fleuve et de sa plaine inondable. Le rapport de la mission interministérielle réalisé à la suite de ces crues, a souligné l'urgence d'engager une vaste réflexion au niveau du bassin pour modifier les pratiques actuelles d'occupation des sols dans les zones inondables et revoir les moyens de protection des zones habitées contre les inondations.
Le 4 août 1994, M. Michel Barnier, ministre de l'Environnement, demandait au Préfet coordonnateur de bassin, d'engager dans le cadre du comité de bassin et de l'Agence de l'Eau, une "étude globale sur les crues du Rhône". Enfin, en décembre 1995, Madame Corinne Lepage, ministre de l'Environnement, souhaitait que la maîtrise d'ouvrage de cette étude soit confiée à un établissement public regroupant l'ensemble des départements riverains du Rhône.

A la demande des Conseils généraux des 11 départements riverains du fleuve Rhône, l'institution Interdépartementale des bassins Rhône Saône (IRS) a été désignée comme maître d'ouvrage d'ensemble de l'étude. Un comité technique associant les collectivités territoriales, les services de l'Etat, le concessionnaire et les associations riveraines, a été mis en place. 


\section{U UN FLEUVE TRÈS AMÉNAGÉ}

Long de $522 \mathrm{~km}$, le Rhône, dans sa partie française, constitue l'artère principale d'échanges de la vallée. Le fleuve est soumis à une forte pression d'usage avec plus de 2.5 millions d'habitants riverains. Il est utilisé pour l'alimentation en eau potable, la production industrielle, la navigation, l'irrigation, la production d'électricité, l'évacuation des eaux usées et les loisirs (baignades, pêche, tourisme). C'est aussi un formidable réservoir d'habitats et d'espèces, tant animales que végétales, $[2,6,9]$. L'une des caractéristiques du fleuve est d'être alimenté sur tout son parcours par de puissants affluents aux réactions parfois brutales.

Le Rhône appartient au domaine public de l'Etat. La police des eaux (prélèvements, rejets, pêche, zone inondable) est assurée par le service navigation Rhône Saône. L'annonce des crues est partagée entre le service de navigation Rhône Saône pour le fleuve compris entre la frontière suisse et Montélimar et la DDE du Vaucluse en aval de Montélimar jusqu'à la mer.

La loi du 27 mai 1921 a permis l'aménagement du fleuve entre la Suisse et la Méditerranée. La Compagnie Nationale du Rhône, créée en 1933, a en charge la production d'énergie électrique, le développement de l'agriculture, le développement de la voie navigable à grand gabarit. La CNR est une "société anonyme d'intérêt général". Son capital de 36 millions de F en 1998 est réparti aux deux tiers sur 317 collectivités riveraines, plusieurs syndicats et des chambres consulaires et pour le tiers restant sur la SNCF et EDF. Elle est donc très liée aux collectivités locales, tout en étant tributaire de l'EDF pour l'achat de sa production électrique. Il est possible que cette situation évolue dans le contexte de la mise en application par la France de la Directive européenne sur l'électricité à l'automne 1999.

Les 18 aménagements hydrauliques de la CNR comportent un barrage et une centrale. Ils sont complétés en aval de Lyon par une écluse à grand gabarit. Cet équipement permet de produire 16 milliards de $\mathrm{kWh}$ par an, soit $4 \%$ de la production électrique française. A partir de prises d'eau et de canaux, la CNR assure un débit global d'environ $100 \mathrm{~m}^{3} / \mathrm{s}$, ce qui permet l'irrigation de 120000 ha agricoles. En 1997, 3,6 millions de tonnes ont été transportées par voie d'eau. Pour la partie du fleuve à l'aval de Lyon, les surfaces inondées avant les aménagements ont été estimées à 42350 ha et celles qui sont pro- tégées par les mêmes aménagements sont de 41220 ha (13 740 ha "totalement" protégés et 27750 partiellement). Cette réduction des zones inondables du fleuve accélère, sans doute, les temps d'écoulement des crues moyennes [3, 4].

Les effets induits de ces aménagements, au-delà de la réduction des zones inondables, se font sentir également sur la dynamique des crues et sur l'équilibre naturel du lit et les niveaux de nappe dans les tronçons court-circuités.

Le SDAGE Rhône Méditerranée Corse, approuvé par le Comité de bassin en 1996 reconnaissant le Rhône comme milieu structurant à l'échelle du bassin, lui assignait un certain nombre d'orientations et demandait notamment la restauration prioritaire de certains tronçons court-circuités du fleuve Rhône ainsi que la réhabilitation des axes de migration des poissons amphihalins [5].

\section{II — UNE VOLONTÉ AFFIRMÉE DE GÉRER GLOBALEMENT L'HYDROSYSTÈME}

Depuis une décennie, plusieurs démarches de planification visant à mieux gérer le fleuve dans toutes les composantes de son hydrosystème ont été engagées. Depuis son adoption par le Comité de Bassin en décembre 1996, le SDAGE Rhône Méditerranée - Corse constitue la base de référence raisonnée et harmonieuse de toute réflexion sur la restauration du Rhône.

La révision du Plan d'Action Rhône, adopté en 1992, doit permettre de rassembler et synthétiser les réflexions conduites sur l'ensemble des thèmes. Il s'agit de ceux qui ont déjà été abordés par le plan d'action antérieur (qualité de l'eau, restauration physique...) pour lesquels des ajustements sont à apporter (révision des objectifs de qualité), des axes de réflexion nouveaux impulsés par le SDAGE (radio-écologie, gestion quantitative de la ressource) ou par les priorités actuelles des différents ministères (gestion des crues et de l'espace alluvial, plan décennal de restauration hydraulique et écologique du fleuve, modernisation de l'annonce des crues).

En 1999, à la demande du Gouvernement, un programme de restauration hydraulique et écologique du fleuve Rhône a été défini par le Préfet coordonnateur de bassin. Pour l'élaboration de ce programme, le Préfet coordonnateur de bassin s'est appuyé sur un comité de pilotage comprenant la Délégation de bassin Rhône-Méditerranée-Corse, l'Agence de l'eau, la C.N.R., EDF, les services de l'état, autorités de

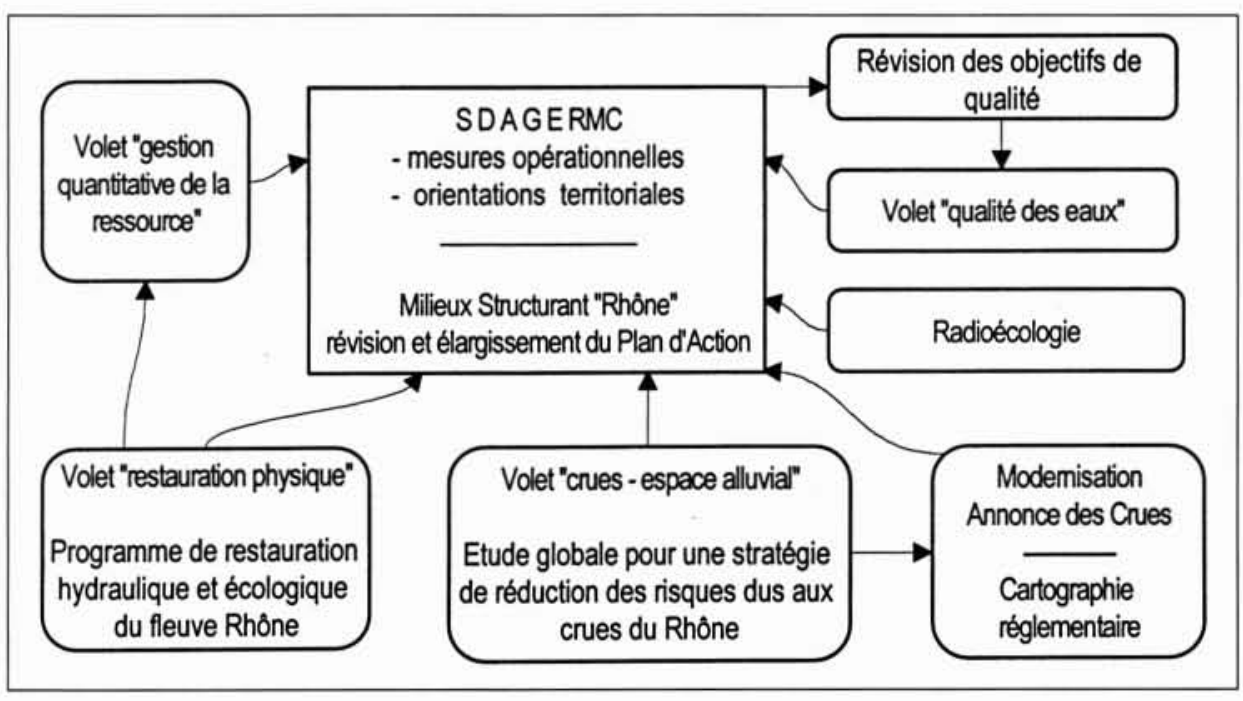

1. Positionnement de l'étude globale pour une stratégie de réduction des risques dus aux crues. 
tutelle de la CNR, des élus impliqués dans la restauration écologique du fleuve, des associations riveraines du Rhône (protection de la nature et pêche), le Conseil Supérieur de la Pêche et les DIREN Provence Alpes Côte d'Azur et Languedoc Roussillon.

Ce programme a été validé par les ministères commanditaires le 2 juin 1999. Le conseil d'administration de l'Agence de l'eau a délibéré favorablement quant au principe de son cofinancement le 17 juin 1999 et le Comité de bassin a soutenu sa mise en œuvre le 2 juillet 1999. La CNR a été appelée par le Gouvernement à contribuer au financement de ce programme à hauteur de $35 \mathrm{MF}$ par an pendant 10 ans $[6,7,10]$. Ce programme constitue le volet "restauration hydraulique et écologique" du plan d'action Rhône révisé.

Quant à l'étude globale pour une stratégie de réduction des risques dus aux crues, elle constitue la composante "gestion de l'espace alluvial" du Plan d'Action Rhône. Son coût estimatif global est de $20 \mathrm{MF}$, dont $10 \mathrm{MF}$ correspondant à l'estimation de la prestation CNR (modélisation hydraulique).

Le schéma 1 illustre le positionnement de l'étude globale et son articulation avec le SDAGE.

\section{III — UNE RÉACTION RAPIDE DE L'ÉTAT CONCERNANT LES CRUES}

A la suite des inondations de 1993 et 1994 qui ont occasionné pour la seule crue d'octobre 1993 des dégâts estimés à plus de 1 milliard de francs de dommages pour une superficie inondée de plus de $300 \mathrm{~km}^{2}$ (cf chronique des crues récentes sur le Rhône reportée en annexe), la mission interministérielle de mars 1994, pilotée par l'Ingénieur général Dambre [1], concluait : "L'ampleur des dégâts ne peut être imputée au dispositif d'annonce des crues, même si celui-ci doit être amélioré et modernisé. Les causes des conséquences dramatiques de ces crues doivent être cherchées ailleurs. Elles résident principalement dans le fort développement économique, agricole et industriel, et l'urbanisation dans les zones inondables, qui n'a que très partiellement pris en compte le risque d'inondation et dans un dysfonctionnement des systèmes de protection contre les crues insuffisamment entretenus, à l'exception de ceux de la CNR" .

Par ailleurs la mission formulait plusieurs remarques importantes, dont deux, reprises ci-dessous, concernant : - la volonté "d'engager une vaste réflexion au niveau du bassin pour modifier les pratiques actuelles d'occupation des sols et renforcer les dispositifs de protection des zones urbanisées ";

- la proposition de création "d'une structure de coopération sur l'ensemble du bassin du Rhône pour favoriser une approche globale".

Dans ce contexte, le 4 août 1994, M. Michel Barnier, ministre de l'Environnement, demandait au Préfet coordonnateur de bassin d'engager, dans le cadre du comité de bassin et de l'Agence de l'Eau, une "étude globale sur les crues du Rhône".

Dès le début de l'année 1995, un secrétariat technique animé par la Délégation de bassin Rhône Méditerranée Corse et constitué de l'Agence de l'eau et du Service Navigation Rhône-Saône, élaborait alors, pour le compte du préfet coordonnateur de bassin et du président du Comité de bas- sin, la trame générale et les projets de cahiers des charges de l'étude globale des crues du Rhône.

Enfin, sensible au vœu formulé en septembre 1995 par le Comité de bassin que cette étude globale implique étroitement les collectivités riveraines, par lettre du 7 décembre 1995, Madame Corinne Lepage, ministre de l'Environnement, souhaitait que la maîtrise d'ouvrage de cette étude soit confiée à un établissement public regroupant l'ensemble des départements riverains du Rhône.

\section{IV - UNE INNOVATION POUR LA MAÎTRISE D'OUVRAGE}

Sollicités par les préfets de région en vue de mettre en place une structure de coopération à l'échelle du fleuve, les présidents de conseils généraux des départements concernés ont d'abord hésité de s'engager dans une démarche pour laquelle ils n'ont pas de compétence définie. La perspective d'un cofinancement des travaux découlant des conclusions de l'étude a sans doute aussi contribué à cette hésitation.

Sous l'impulsion du Préfet Bernard, coordonnateur de bassin, considérant l'importance des enjeux et sensibles à l'aspect novateur de la démarche pour une gestion globale et solidaire du fleuve et de la zone inondable, les Départements ont finalement jugé nécessaire la réalisation de l'étude globale des crues du Rhône et se sont engagés à participer à la structure de coopération et au financement de l'étude.

L'Institution Interdépartementale des Bassins Rhône Saône, l'IRS, est désignée comme maître d'ouvrage de l'étude, la partie hydraulique relevant des obligations du concessionnaire est à la charge et sera réalisée par la CNR. Pour une totale transparence, I'IRS a créé un budget annexe pour cette mission.

Un comité de coordination animé par le Président du Comité de Bassin et le Préfet de Région coordonnateur de bassin est instauré. Le comité devra assurer la coordination indispensable entre les différents acteurs et partenaires de l'étude et également entre les grandes options de l'étude et les orientations du SDAGE spécifiques au fleuve Rhône.

Pour ce cadrage institutionnel innovant, I'IRS a dû changer ses statuts et signer une convention spécifique avec les onze Conseils Généraux riverains du fleuve Rhône. Lors d'une réunion entre les présidents des conseils généraux, les préfets. le président du comité de bassin, organisée le 22 septembre 1997 par le Préfet Besse, coordonnateur de bassin, le schéma général de fonctionnement a été confirmé et notamment l'articulation entre ce projet et les autres actions menées sur le Rhône (SDAGE, Plan d'Action Rhône, rôle de l'Etat sur les risques et l'annonce de crues, rôle et missions de la CNR).

Il aura donc fallu près de 2 années de négociations entre l'Etat, I'IRS et le Comité de bassin pour aboutir à ce dispositif.

\section{CONTENU DE L'ÉTUDE GLOBALE}

L'étude porte sur le fleuve allant de la frontière suisse à la mer. Son objectif principal est de définir une stratégie de réduction des risques dus aux inondations. Pour ce faire, une analyse des conséquences des modifications apportées ces cinquante dernières années au réseau hydrographique sera nécessaire. Par ailleurs, l'analyse devra fournir des recommandations pour la mise en œuvre d'une gestion de l'occupation des sols intégrant mieux le risque inondation. Des informations sont également attendues, afin d'améliorer le 


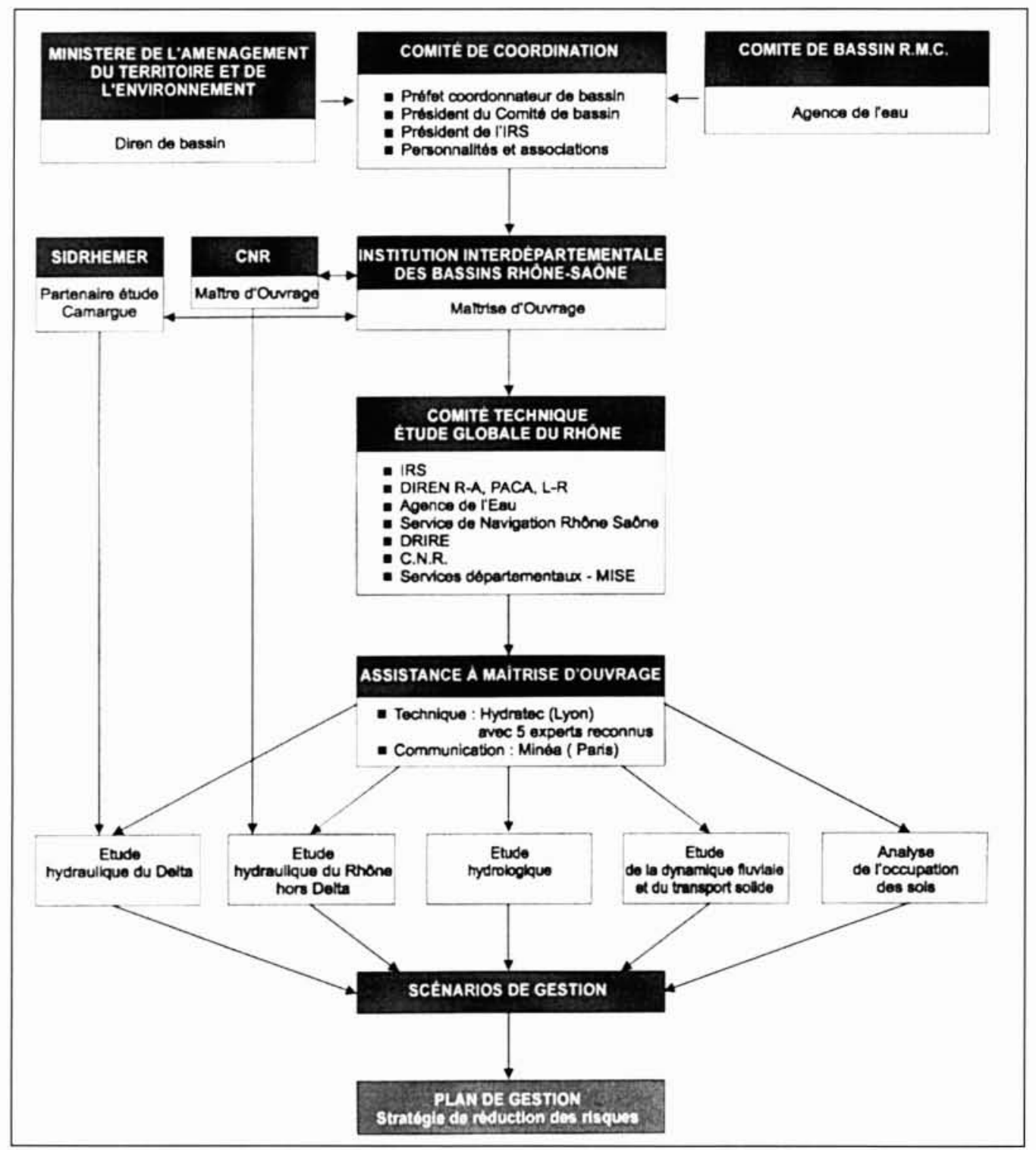

2. Organigramme
fonctionnel de l'étude. système d'alerte des crues sur le fleuve Rhône (notamment en termes de connaissance des temps de propagation des crues et de cotes de déversement aux points critiques).

Les différents volets de l'étude sont les suivants :

- analyse de l'hydrologie des apports de crue,

- modélisation hydraulique des crues fortes à très fortes du fleuve y compris dans sa zone deltaïque,

- analyse de la dynamique fluviale et du transport de matériaux,

- analyse de l'occupation des sols et des impacts socioéconomiques dus aux inondation.

A l'issue de ces différentes phases, des scénarios de gestion et d'aménagement visant à réduire les risques liés aux crues seront proposés. Ces scénarios feront l'objet d'une concertation avec les riverains du fleuve.

\subsection{Analyse hydrologique}

Maurice Pardé a réalisé en 1925 la dernière étude hydrologique d'ensemble sur le Rhône. Ce travail, qui a été complété en 1942, malgré sa très grande qualité et compte tenu des aménagements multiples sur le bassin du Rhône, dont ceux de la CNR effectués depuis, nécessite d'être actualisé.

L'étude se propose, à partir de l'analyse des crues d'importance majeure observées depuis le début du siècle (février 1928, novembre 1935, novembre 1944 , novembre 1951 , octobre 1993, janvier 1994, etc.) et des développement méthodologiques récents en hydrologie statistique, d'approfondir les connaissances sur la genèse des crues et de définir des scénarios de crues fortes à exceptionnelles qui prennent en compte la complexité des mécanismes météorologiques caractérisant le bassin versant du Rhône.

\subsection{Modélisation du Rhône hors delta}

La CNR, au titre de sa concession, est chargée de la réalisation de ce volet de l'étude. Elle mettra en œuvre le modèle mathématique existant de propagation des crues du Rhône de la CNR dans la partie concédée du fleuve entre le barrage de Chancy-Pougny et la restitution de Vallabrègues. Le modèle est de type mono-dimensionnel à casiers. Il s'accompagne d'une analyse cartographique des casiers inondables, issus de la conception historique de l'aménagement de la vallée, dite "Architecture du modèle" qui doit être présentée 
à l'ensemble des conseils généraux pour valider si toutes les particularités locales ont été correctement prises en compte. Cette phase de concertation est capitale pour permettre de la part des élus une bonne compréhension de la démarche, des résultats obtenus lors de la progression de l'étude et, en conséquence, leur participation active à l'élaboration des scénarios de gestion.

Le modèle simule les conditions de propagation des crues dans le lit endigué du Rhône, dans les ouvrages de dérivation et dans la plaine alluviale située derrière les digues. Il prend également en compte les principaux affluents dans les zones sous influence des aménagements du Rhône.

\subsection{Etude de la dynamique fluviale et du transport solide}

Le Rhône autrefois "libre" était un fleuve très mobile qui transportait une importante charge solide comme en témoigne l'importance du Delta. Les endiguements réalisés au XIX ${ }^{\mathrm{c}}$ siècle, les aménagements "Girardon", puis de façon plus notable, les aménagements hydroélectriques et les extractions de granulats dans le lit du fleuve et ses principaux affluents, la construction de seuils ont entraîné de profondes modifications dans la morphologie et le transit solide du fleuve. Les apports du Rhône à la mer pourraient avoir très sensiblement diminué. Les conséquences peuvent se ressentir y compris sur les parties meubles du littoral méditerranéen. De graves problèmes de tenue des digues de protection et la menace d'une estuarisation du delta peuvent en découler.

L'étude analysera les modifications observées dans le régime de transport solide du Rhône et débouchera sur des propositions d'actions visant à rétablir l'équilibre sédimentaire dans les zones actuellement perturbées (vieux Rhône, Camargue, etc.).

\subsection{L'occupation des sols et la synthèse environnementale}

Le recensement des enjeux dans le lit du fleuve, qu'ils soient sociaux, économiques, environnementaux ou patrimoniaux, permettront d'évaluer les dommages potentiels selon l'importance de la crue. Mis face à face avec les coûts des aménagements nécessaires à une relative réduction des risques, ils orienteront les choix des responsables rhodaniens. L'étude décrira dans un premier temps les enjeux dans les zones inondables compte tenu de l'occupation des sols actuelle. Elle estimera ensuite les impacts et les dommages dus aux inondations dans la situation actuelle et pour différents scénarios de gestion et d'aménagement de la vallée.

\subsection{Modélisation du delta}

L'étude s'inscrit dans la continuité de l'étude hydraulique menée par la CNR. Elle répond aux résultats de la mission interministérielle d'avril 1999 conduite par Monsieur l'ingénieur général Pierre Balland sur la définition des travaux de protection contre les inondations en Camargue [12]. Elle redéfinira l'aléa hydraulique dans la zone deltaïque lié aux différents scénarios possibles de crues du Rhône et de tempêtes marines et définira les fondements d'une stratégie globale de protection contre les inondations. Elle s'appuiera sur le confortement optimisé des digues (protection des zones urbaines) et la gestion contrôlée des flux à l'intérieur de l'île.

Elle s'appuiera sur une modélisation hydraulique couvrant toute la zone deltaïque historiquement inondable en aval de Beaucaire. L'architecture du modèle sera soumise à concer- tation. Elle sera conçue pour simuler différentes configurations possibles d'aménagement du delta.

\subsection{Elaboration des scénarios de gestion et d'aménagement et définition d'une stratégie de réduction des risques.}

A l'issue des premières phases des études, une synthèse de l'état initial sera rédigée. Elle précisera le régime des apports de crue, les conditions d'écoulement et de propagation de ces crues dans le corridor fluvial, les dysfonctionnement actuels dans le bilan sédimentaire du fleuve et leurs conséquence en matière d'évolution du lit et des milieux physiques et biologiques associés et caractérisera les risques socio-économiques liés aux crues.

Après prise de connaissance de ces résultats par l'ensemble des partenaires, des scénarios de gestion et d'aménagement seront proposés. Les différents scénarios envisageables qui feront l'objet d'une simulation par les modèles pourront porter sur :

- la réouverture d'anciens champs d'expansion de crue ;

- la modification de la gestion en crue des ouvrages CNR ;

- la modification de la gestion des ouvrages EDF sur les affluents ;

- la modification ou le renforcement des digues :

- des recommandations en matière de gestion de l'espace et de protection des zones densément habitées ;

- le compartimentage de certaines zones situées derrière les digues :

- l'entretien et la restauration du lit dans le vieux Rhône.

\section{VI $\square$ COMMUNIQUER POUR AIDER À MIEUX DÉCIDER}

L'Institution interdépartementale des bassins Rhône-Saône a introduit, dès le démarrage des études, une démarche "communication". La volonté des élus est d'accompagner les bureaux d'études chargés des recherches techniques en leur donnant des orientations respectueuses des riverains et un souci d'explication de leur travail en cours. Réciproquement, I'IRS souhaite mobiliser les responsables locaux sur les enjeux et sensibiliser les décideurs au comportement à tenir par rapport aux problématiques de vulnérabilité. La dimension même du partenariat avec onze départements et plusieurs administrations plaide aussi en faveur d'une transparence et d'une éthique d'ouverture sur un sujet complexe et peut-être dérangeant.

En ce qui concerne le déroulement de la mission communication, le volet fait partie des missions techniques et se lie complètement à la progression de l'étude. Pour le démarrage, il a été décidé de faire un tour de table des onze départements pour procéder à un recueil des éléments à introduire dans les cahiers des charges des appels d'offres. Parallèlement, une première série d'interviews de dirigeants d'associations ou de responsables de sociétés de protection de la nature a été mise en œuvre avec pour objectif, là aussi, un recueil des attentes en matière de gestion du fleuve en crue. Pour une large distribution, un dossier clair et suffisamment consistant a été élaboré concernant le contenu des études afin de permettre des réactions et un mûrissement préalable. En ce qui concerne la modélisation, il a été demandé à la CNR de réaliser une cartographie très compréhensible et de procéder à un tour de table sur "l'architecture du modèle" pour à la fois sensibiliser les 
responsables sur l'ampleur des zones inondables et vérifier que le "terrain" est concrètement pris en compte.

En ce qui concerne l'architecture du modèle hydraulique de la CNR, celle-ci a fait l'objet d'une présentation détaillée aux différents partenaires de l'étude et aux acteurs locaux du Rhône (les maires de l'ensemble des communes riveraines, les conseillers généraux, les services de l'Etat, les services techniques des villes et des Départements, les associations...) lors de cinq réunions organisées par l'IRS en décembre 1999, janvier et février 2000, couvrant l'ensemble du cours du fleuve.

Outre les remarques faites en réunions, il a été convenu, à la demande de M. Monteil, président de I'IRS, que chaque commune riveraine et service technique de l'Etat soit destinataire de cartes au 1/25 000 sur lesquelles figureront explicitement les différents casiers et leur fonctionnement tel que prévu initialement par la CNR.

Il n'aura pas échappé aux communes, à leurs services techniques, mais aussi aux services des collectivités ou de l'Etat gestionnaires d'infrastructures (routes, digues, réseaux...), notamment dans les grandes agglomérations urbaines pour lesquels les enjeux humains et économiques sont élevés, sollicités systématiquement par l'IRS, que de la validation de l'architecture du modèle CNR dépendra, en partie, la fiabilité des résultats. C'est d'ailleurs pourquoi le délai de réponse d'une telle consultation a été fixé à un mois. A l'issue de cette consultation ayant associé l'ensemble des collectivités riveraines du Rhône, il est prévu que la synthèse des remarques soit effectuée par CNR et IRS, et que l'architecture du modèle soit modifiée en conséquence. Cette synthèse, comprenant également les remarques issues des partenaires pour le Rhône camarguais, devrait être réalisée pour fin mars 2000 .

Une démarche similaire sera envisagée, en ce qui concerne la cartographie de l'occupation des sols, de manière à évaluer, en concertation, les impacts socio- économiques dus aux inondations.

\section{VII - CONCLUSION}

L'étude globale pour une stratégie de réduction des risques dus aux crues du Rhône telle qu'elle va maintenant s'engager, portée par l'IRS, va constituer à coup sûr pour les riverains du Rhône une démarche remarquable à plusieurs titres.

Tout d'abord, elle se fera dans un contexte affirmé de gestion globale de l'hydrosystème, dans le droit fil des préconisations du SDAGE Rhône - Méditerranée - Corse approuvé par le Comité de bassin en 1996. La gestion stricte de l'eau au plan purement hydraulique est dépassée, c'est l'ensemble de l'espace alluvial qui est concerné, de la frontière suisse à la Méditerranée, y compris l'île de la Camargue. Les compartiments suivants seront abordés : hydrologie, hydraulique, transports solides, occupation des sols.

Les aspects socio-économiques seront pris en compte dans le cadre d'une démarche interactive devant déboucher sur des propositions de scénarios de gestion de l'espace alluvial permettant de limiter au mieux le risque lié aux grandes inondations. C'est pourquoi le volet communication fait intimement partie des missions techniques et est lié à la progression de toutes les phases de l'étude.

Cette démarche se déroule dans un contexte clairement affiché par les Départements riverains du fleuve, dont l'IRS est l'émanation, de gestion concertée et solidaire des risques liés aux grandes inondations du fleuve.
Elle devrait, une fois aboutie, constituer un outil décisionnel performant en matière d'occupation du territoire à disposition des collectivités et des services de l'Etat (POS hydraulique à l'échelle du bassin versant), répondant en cela à l'objectif principal assigné à l'étude par la mission présidée par l'ingénieur général Dambre en 1994 : "modifier les pratiques actuelles d'occupation des sols dans les zones inondables et revoir les moyens de protection des zones habitées contre les inondations."

Cette démarche devrait également permettre aux riverains d'accéder à la compréhension des phénomènes de genèse et de propagation des grandes crues sur le Rhône, de participer à l'élaboration et de retenir des scénarios solidaires de gestion du risque inondation à l'échelle du fleuve dans son ensemble, scénarios eux-mêmes issus de scénarios hydrologiques croissants dans leur gravité et leur ampleur à l'échelle du fleuve et à la définition desquels ils auront été étroitement associés.

Enfin, dans un contexte où, par suite de l'augmentation du taux de $\mathrm{CO}_{2}$ dans l'atmosphère, des hypothèses de changement global du climat sont maintenant régulièrement évoquées, ce changement climatique risquant d'affecter les régimes hydrologiques, ce qui, sous réserve de confirmation, pourrait se traduire par une exacerbation des extrêmes (crues, étiages), [13, 14], il ne fait pas de doute que les collectivités riveraines du fleuve et les gestionnaires responsables suivront avec une attention toute particulière la progression et les résultats de cette étude.

\section{Références}

[1] Mission interministérielle sur les inondations de la vallée du Rhône, en aval de Lyon d'octobre 1993 et de janvier 1994. Rapport de synthèse Mai 1994.

[2] Les espaces naturels de la vallée du Rhône : éléments pour une politique de gestion intégrée : J.L Michelot - thèse Doct. Lyon III, 523 pages : 1989

[3] Incidence de l'aménagement du lit du Bas Rhône sur la propagation des crues : Mathian, Société hydrotechnique de France ; Paris, 1968

[4] Les crues du Rhône, d'octobre 1993 et janvier 1994 : CNR - Direction de l'exploitation, mars 1994.

[5] SDAGE Rhône Méditerranée Corse ; Comité de bassin, décembre 1996 ; vol. 1: La vallée du Rhône et son aménagement ; vol. 3 : carte 7 , carte 10 , carte 11 : vol. 2 : p. 76-77 : " Législation garantissant la libre circulation des espèces piscicoles ".

[6] Programme décennal de restauration hydraulique et écologique du fleuve Rhône - Diren Rhône - Délégation de bassin : Agence de l'eau RMC - Avril 1999.

[7] Impact des aménagements hydroélectriques sur l'écosystème Rhône : Monique Coulet, Béatrice Venard, Philippe Monnet FRANA Lyon, Région Rhône-Alpes, Agence de l'eau RMC, 1997.

[8] Modernisation de l'annonce des crues sur le Rhône (du Léman à la Méditerranée). Besoin de modernisation Diren Rhône Alpes - délégation de bassin RMC, janvier 1998.

[9] Inondations en Languedoc - Roussillon des 12-13/11/1999 ; Note sur les premières propositions techniques et organisationnelles ; réflexions et enseignements pour l'annonce des crues. Préfet coordonnateur de bassin, Diren Rhône Alpes Délégation de bassin : Janvier 1999.

[10] Dérives écologiques et gestion du milieu fluvial rhodanien. J. F. Fruget : J.L. Michelot : in Revue géographique de Lyon : vol. 72-nol, 1997 Le Rhône l'axe et sa vallée. p. 35-48.

[11] Le Rhône entre nation et région : Jacques Béthemont : in Revue géographique de Lyon ; vol. 72-no 1,1997 p 67-75.

[12] Rapport sur la gestion de l'eau en Camargue : la protection contre les inondations ; Pierre Balland et al. ; Conseil général des Ponts et Chaussées : Mission d'inspection Spécialisée de l'Environnement : Affaire no 1998-0250-01-A : Paris la Défense, le 3 Mai 1999. 
[13] Modélisation hydrologique à l'échelle régionale appliquée au bassin du Rhône. Comparaison de deux modes de calcul des bilans hydriques de surface et étude de sensibilité à une perturbation des forçages climatiques. C. Golaz-Cavazzi, Thèse ENS Mines de Paris : 16 Septembre 1999.

[14] Programme du MATE, gestion des impacts du changement climatique : en cours : "étude des impacts potentiels du changement climatique sur le bassin versant du Rhône en vue de leur gestion. BRGM-CEMAGREF-EDF-ENSMP-Météo-France ".
[15] Rapport de M. le Député Yves Dauge, Maire de Chinon, conseiller général d'Indre et Loire, sur les politiques de prévention des inondations : 19 Novembre 1999.

\section{Annexe : Chronique des crues récentes sur le Rhône}

Par ses caractéristiques (relief, hydrographie, climatologie), le bassin du Rhône est sans doute le plus exposé des bassins fluviaux français aux risques des inondations. Le Rhône est le fleuve de France qui a les plus fortes crues. Le débit de la crue millénaire à Beaucaire est estimé à $14000 \mathrm{~m}^{3} / \mathrm{s}$. En outre, les affluents à régime cévenol ou alpin peuvent avoir des crues importantes et dévastatrices en quelques heures. Les années 1990 ont connu de fortes crues sur l'ensemble du cours du Rhône français alors que pendant près de 40 ans les riverains n'avaient pas eu à faire face à des inondations d'une telle ampleur.

Au début du siècle, le Haut Rhône a été touché par plusieurs crues importantes et rapprochées : 1910, 1914 et 1918. Puis, les crues de 1928 et 1944 ont été suivies d'une longue période de calme jusqu'en février 1990 qui a particulièrement touché la Chautagne et les communes de Brangues, le Bouchage, les Avenières dans le Département de l'Isère. Mais si l'on consulte la chronique des crues à Bognes, en aval de Génissiat, depuis 1853 , c'est très caractéristique : sur les 10 crues les plus importantes, 6 appartiennent à la seconde moitié du siècle dernier.

La crue de février 1990 fait partie des crues historiques sur le Haut-Rhône. A partir du 11 février 1990, une perturbation climatique de régime Nord-Ouest touche le bassin. Elle provoque des chutes de neige importantes jusqu'à $400 \mathrm{~m}$ d'altitude, notamment les 12 et 13 février. Dans la nuit du 13 au 14 , un régime de vent du sud-ouest s'établit. Il entraîne une forte augmentation de la température, remontant celle-ci jusqu'à $0^{\circ}$ à $2200 \mathrm{~m}$ d'altitude. Selon les secteurs, pendant 48 à 72 heures, des pluies continues et abondantes se succèdent et font fondre la neige fraîche sur l'Ain, les Savoies et les massifs montagneux de l'Isère. Ces précipitations atteignent ou dépassent $200 \mathrm{~mm}$ pour une durée de 30 heures en Savoie, de 48 heures dans l'Ain et de 72 heures dans le Nord Isère. Les lits des cours d'eau font face, en deux jours, à l'évacuation d'une quantité d'eau voisine du double de la précipitation normale mensuelle ! La réaction des rivières est immédiate. Les gradients de montée sont très importants, tant sur le Rhône que sur les affluents. Sur le Rhône, la croissance du débit atteint $200 \mathrm{~m}^{3} / \mathrm{s}$ par heure ! Les grandes zones d'écrêtement des crues sont fortement sollicitées. Ainsi le lac du Bourget monte de $2,45 \mathrm{~m}$ environ au-dessus de son niveau normal en seulement 4 jours. Les plaines inondables, notamment Brangues et le Bouchage, sont submergées..

Plus récemment, sur le Bas Rhône, les deux grandes crues de 1993 et 1994 peuvent avoir surpris par leur importance et leur proximité dans le temps. Cependant, les chroniques tenues depuis la seconde moitié du XIX ${ }^{\mathrm{e}}$ siècle nous rappellent que des événements semblables ont déjà eu lieu. Sachant que le régime moyen du Rhône est à Viviers de $1500 \mathrm{~m}^{3} / \mathrm{s}$ et à Beaucaire de $1700 \mathrm{~m}^{3} / \mathrm{s}$, l'hiver 1935 - 1936, par exemple, a connu deux grandes crues avec, en novembre, $6000 \mathrm{~m}^{3} / \mathrm{s}$ à Viviers et
$9600 \mathrm{~m}^{3} / \mathrm{s}$ à Beaucaire puis, en janvier, $5800 \mathrm{~m}^{3} / \mathrm{s}$ à Viviers et $7800 \mathrm{~m}^{3} / \mathrm{s}$ à Beaucaire.

La crue de septembre - octobre 1993 est caractérisée par une série d'épisodes pluvio-orageux qui se succèdent à partir du 6 septembre. Du 6 au 15 septembre, des pluies abondantes affectent le rebord cévenol et remontent au Nord. Un second épisode orageux, localement très violent, se généralise du 22 au 25 septembre jusqu'à la partie septentrionale du bassin. Le 22 septembre, deux noyaux pluvieux très importants se développent, le premier sur les Cévennes avec plus de $300 \mathrm{~mm}$ de précipitations en 24 heures, le second en Provence sur l'axe Aix-en-Provence - Vaison-la-Romaine. Ces deux épisodes successifs saturent les sols sur l'ensemble du bassin rhodanien et créent une première onde de crue sur la partie aval du Rhône avec, à Beaucaire, un maximum de $6700 \mathrm{~m}^{3} / \mathrm{s}$ le 25 septembre. Dans la nuit du 30 septembre au $I^{\text {er }}$ octobre, de violents orages se déchaînent sur le Nord Vaucluse et provoquent la crue du Lez qui submerge la ville de Bollène. Le Rhône roule alors de fortes eaux supérieures à la crue annuelle, lorsqu'une averse méditerranéenne généralisée affecte le bassin du 5 au 8 octobre et provoque une très forte crue sur l'ensemble du cours du Rhône les 8,9 et 10 octobre. A Ternay, le 10 octobre, on observe un débit de $4420 \mathrm{~m}^{3} / \mathrm{s}$, soit une fréquence de retour estimée à 8 ans. Avec les apports du bassin versant intermédiaire et le débit de l'Isère, la crue connaît son maximum à Valence le 8 octobre à 15 heures, $6700 \mathrm{~m}^{3} / \mathrm{s}$, soit une fréquence de retour de 35 ans. La pointe de crue passe à Viviers, le 9 octobre vers 1 heure, avec $7700 \mathrm{~m}^{3} / \mathrm{s}$. Elle correspond alors à une fréquence de retour d'environ 80 ans. C'est la pointe la plus importante observée pour cette crue. Les pluies de fin décembre, amenées par une série de perturbations océaniques, gonflent à nouveau les cours d'eau.

Entre le 3 et le 6 janvier 1994, le débit du Rhône entre Valence et Beaucaire évolue entre 4000 et $4600 \mathrm{~m}^{3} / \mathrm{s}$ lorsque, à nouveau, comme en 1993, un épisode pluvieux de type méditerranéen intéresse le bassin du Rhône et de ses affluents à partir de Valence. Les ondes de ces crues unitaires se cumulent pour donner une pointe à Viviers de $7600 \mathrm{~m}^{3} / \mathrm{s}$ le 7 janvier à 13 heures 30 et $11000 \mathrm{~m}^{3} / \mathrm{s}$ à Beaucaire, le 8 , entre 7 heures et 7 heures 30 , soit une période de retour encore plus rare, de l'ordre de 100 ans pour cette localisation.

Ce n'était pas fini ! La décrue est dans un premier temps rapide, mais elle est stoppée par un nouvel épisode pluvieux dans la nuit du 10 au 11 janvier qui provoque une brutale remontée du débit à Viviers, à Beaucaire et en Camargue.

Ces crues ont occasionné des dégâts considérables. La crue de 1993, dont la période de retour a été estimée à 50 ans, a causé plus d'un milliard de francs de dommages pour une superficie inondée de plus de $300 \mathrm{~km}^{2}$. Elle a rappelé avec force que la vallée du Rhône reste très exposée aux risques d'inondation. 